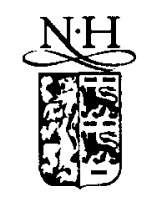

\title{
All solid-state diode pumped Nd:YAG MOPA with stimulated Brillouin phase conjugate mirror
}

\author{
H.L. Offerhaus, H.P. Godfried, W.J. Witteman \\ Nederlands Centrum voor Laser Research B.V., Postbus 2662, 7500CR Enschede, Netherlands
}

Received 24 October 1995; revised version received 7 February 1996; accepted 27 February 1996

\begin{abstract}
At the Nederlands Centrum voor Laser Research (NCLR) a $1 \mathrm{kHz}$ diode-pumped Nd:YAG Master Oscillator Power Amplifier (MOPA) chain with a Stimulated Brillouin Scattering (SBS) Phase Conjugate mirror is designed and operated. A small Brewster angle Nd:YAG slab ( 2 by 2 by $20 \mathrm{~mm}$ ) is side pumped with $200 \mu$ s diode pulses in a stable oscillator. The oscillator is $Q$-switched and injection seeded with a commercial diode pumped single frequency $\mathrm{CW}$ Nd:YAG laser. The output consists of single-transverse, single-longitudinal mode $25 \mathrm{~ns}$ FWHM-pulses at $1064 \mathrm{~nm}$. The oscillator slab is imaged on a square aperture that transmits between 3 and $2 \mathrm{~mJ}$ (at 100 and $400 \mathrm{~Hz}$, resp.) The aperture is subsequently imaged four times in the amplifier. The amplifier is a 3 by 6 by $60 \mathrm{~mm}$ Brewster angle zig-zag slab, pumped by an 80-bar diode stack with pulses up to $250 \mu \mathrm{s}$. After the second pass the light is focused in two consecutive cells containing Freon-113 for wave-front reversal in an oscillator/amplifier-setup with a reflectivity of $60 \%$. The light then passes through the amplifier twice more to produce $20 \mathrm{~W}$ (at $400 \mathrm{~Hz}$ ) of output with near diffraction limited beam quality. To increase the output to $50 \mathrm{~W}$ at $1 \mathrm{kHz}$ thermal lensing in the oscillator will be reduced.
\end{abstract}

\section{Introduction}

Power scaling in Nd:YAG lasers is limited by thermally induced stress, causing wavefront distortions and birefringence. Diode lasers allow for more efficient pumping and in combination with phaseconjugation for wavefront restauration enable power scaling to much higher levels while maintaining a good beam quality. These high power sources can be used to efficiently pump doubling crystals, Optical Parametric Oscillators (OPO's) or to produce X-ray plasmas for photolithography. Stimulated Brillouin Scattering (SBS) as a means for Phase Conjugation was discovered as early as 1965. The importance for high power lasers was not realized until much later [1-4]. SBS has been modeled and measured extensively with respect to fidelity, reflectivity and bandwidth [5-7]. Diode pumped YAG-lasers have also been reported on, showing the characteristic high efficiency of diode pumping $[8,9]$.

\section{Oscillator}

The oscillator was reported on separately [10]. A schematic is shown in Fig. 1. The oscillator crystal is pumped with a 5 bar quasi-CW diode array from Spectra Diode Labs. The duration of the pump pulse is $200 \mu$ s at maximum current. The $2 \times 2 \times 20 \mathrm{~mm}$ YAG-slab is mounted in the vertical plane between two copper blocks for cooling. The slab dimensions were chosen for optimal side pumping and mode vol- 


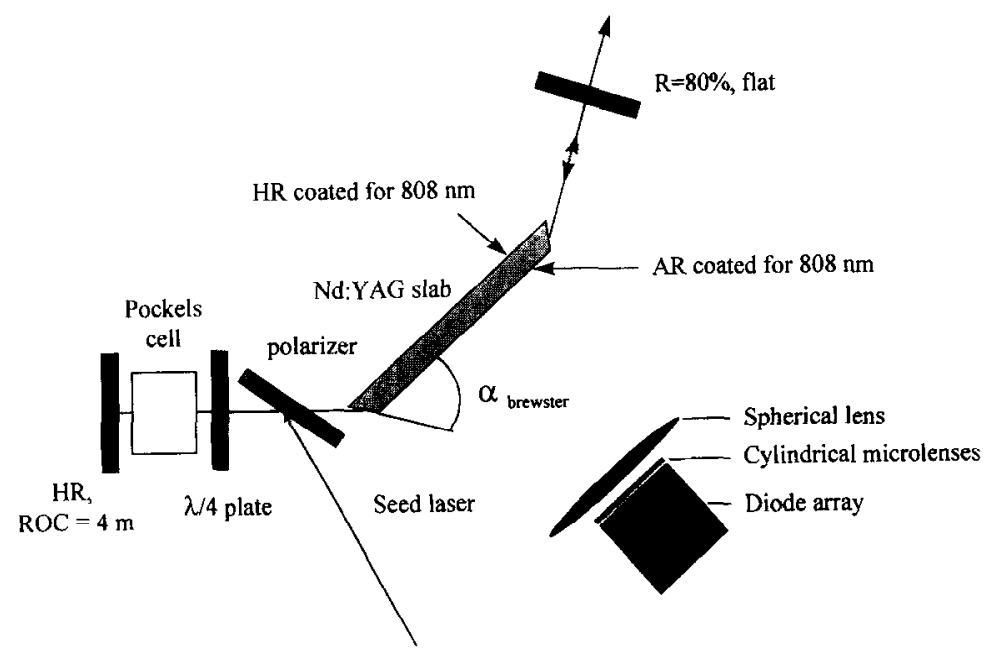

Fig. 1. Schematic of the oscillator.

ume control, with Brewster-angle faces for low-loss transmission without coatings. The injection seeding is done with a commercial single longitudinal mode $\mathrm{CW}$ diode pumped Nd:YAG laser. Since this is a standing wave oscillator the single longitudinal mode output is about $85 \%$ of the multi-mode output. The high reflecting mirror is mounted on a piezo transducer, allowing the cavity length to be adjusted. Initially adjustments were made by manually changing the voltage over the piezo. Typically the cavity would remain locked for up to $10 \mathrm{~min}$. Although this approach was adequate it would constitute a risk for optical damage of components. Therefore an electronic locking mechanism has been built to stabilize the locking. When pumped with pulses of $200 \mu$ s the oscillator produces smooth single longitudinal single transverse mode pulses of $25 \mathrm{~ns}$ FWHM. The (unseeded) output pulse-energy decreases from $3.5 \mathrm{~mJ}$ to $2.5 \mathrm{~mJ}$ as repetition rate increases from $100 \mathrm{~Hz}$ to $400 \mathrm{~Hz}$. This is caused by thermal lensing which decreases the mode size in the slab. This thermal lensing is the limiting factor in this geometry. Since the slab is cooled on opposite sides of an otherwise rectangular geometry, the thermal gradient is predominantly one-dimensional. The lensing is therefore cylindrical. To compensate this lensing a single negative cylindrical lens was introduced in the cavity, allowing laser operation up to $1 \mathrm{kHz}$. Additional non-cylindrical deformations in the slab however caused a degraded phasefront and an unsatisfying

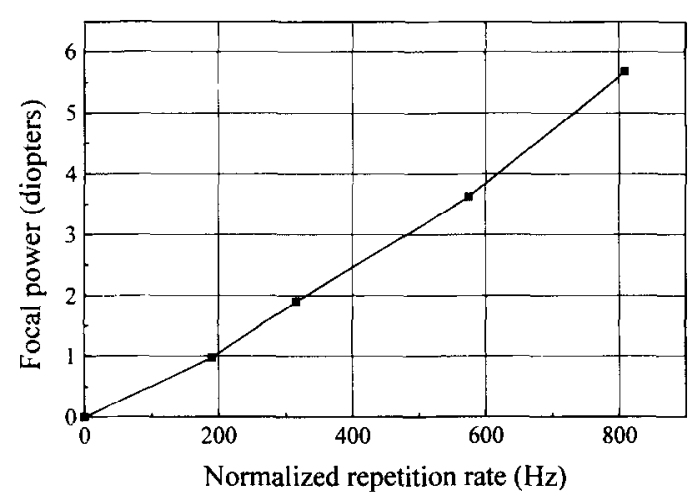

Fig. 2. Thermal lensing of "brick" slab of comparable size.

output. A graph of the lensing as a function of deposited power for a comparable rectangular "brick"slab is given in Fig. 2. In a new design a zig-zag slab is used to compensate this lensing and other phasedeformations, enabling high beam-quality operation up to $1 \mathrm{kHz}$. The cavity configuration is changed to a ring oscillator to extract efficiently in single longitudinal mode.

\section{Relay imaging}

The oscillator output is relay-imaged on a square aperture to adjust the beam cross section to the rectangular amplifier. About $85 \%$ of the energy is transmitted through the aperture and subsequently relay- 


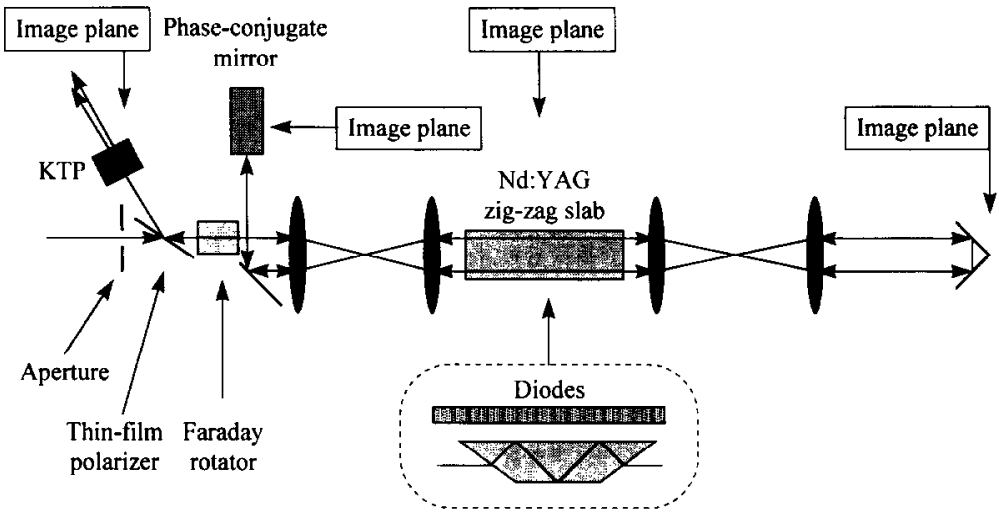

Fig. 3. Schematic of the amplifier chain.

imaged four times onto the amplifier (see Fig. 3). This relay-imaging ensures correct beam propagation through the amplifier slab and avoids high intensity diffraction rings striking the sides of the amplifier. It also reduces diffractive propagation losses, makes the setup less sensitive to beam wander and reduces damage risks in case of thermal lensing. All imaging is done with telescopes so that the beams are collimated and the phase curvature in the image plane is absent. Vacuum cells can be placed to avoid the optical breakdown of air that can occur at the foci in the middle of the telescopes when the laser is operated at high power.

\section{Amplifier}

The amplifier consists of a Brewster-angle zig-zag slab. The zig-zag geometry guards against thermal lensing, stress induced biaxial focusing and birefringence. It is water cooled on the top and bottom face and pumped through the upper face. (The lower face will also be pumped in future experiments.) Used as an oscillator in a short stable cavity it produced 150 $\mu$ s pulses of $68 \mathrm{~mJ}$ at $400 \mathrm{~Hz}$. As an amplifier it has a small signal gain of 3.5 at a stored energy of about $80 \mathrm{~mJ}$.

\section{Stimulated Brillouin scattering}

After double passing the amplifier the light is focused into a set of Brillouin cells for phase-
Brillouin reflectivity for $1064 \mathrm{~nm}$. 20nsec, $D=8 \mathrm{~mm}$ pulses focussed with a $250 \mathrm{~mm}$ lens ( $150 \mathrm{~mm}$ for the second cell)

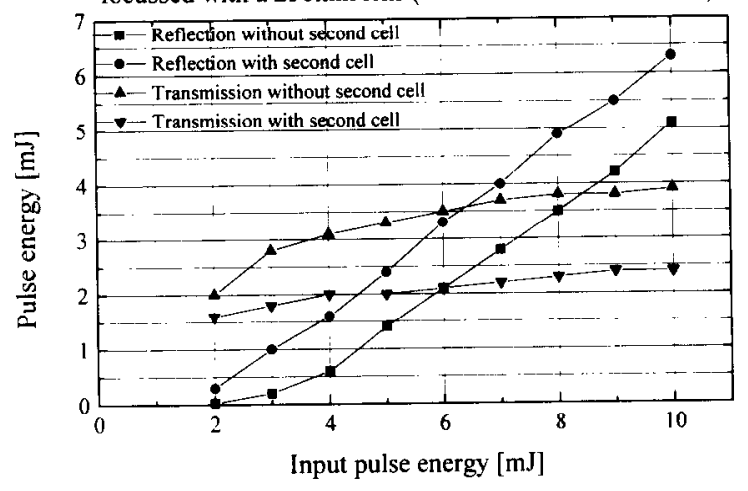

Fig. 4. Brillouin reflectivity measurements with and without a second cell.

conjugation. After the reflection from the Brillouin grating the light passes the amplifier twice more, thereby compensating any phase-front errors introduced by the amplifier. To decrease the threshold and increase the dynamic range of the phase conjugate mirror an oscillator/amplifier configuration is used [11]. The light is first focused with a $200 \mathrm{~mm}$ lens into a $500 \mathrm{~mm}$ cell and then with a $50 \mathrm{~mm}$ lens into a $100 \mathrm{~mm}$ cell. The total length is kept short to avoid pulse compression and self oscillation. Fig. 4 shows results of tests with low rep-rate pulses in a similar setup. These graphs show a slope efficiency of $75 \%$ and a threshold energy of $1.7 \mathrm{~mJ}$. The second cell lowers the threshold but does not alter the slope efficiency. No significant pulse compression is observed. In the MOPA chain an overall reflection of $60 \%$ is 

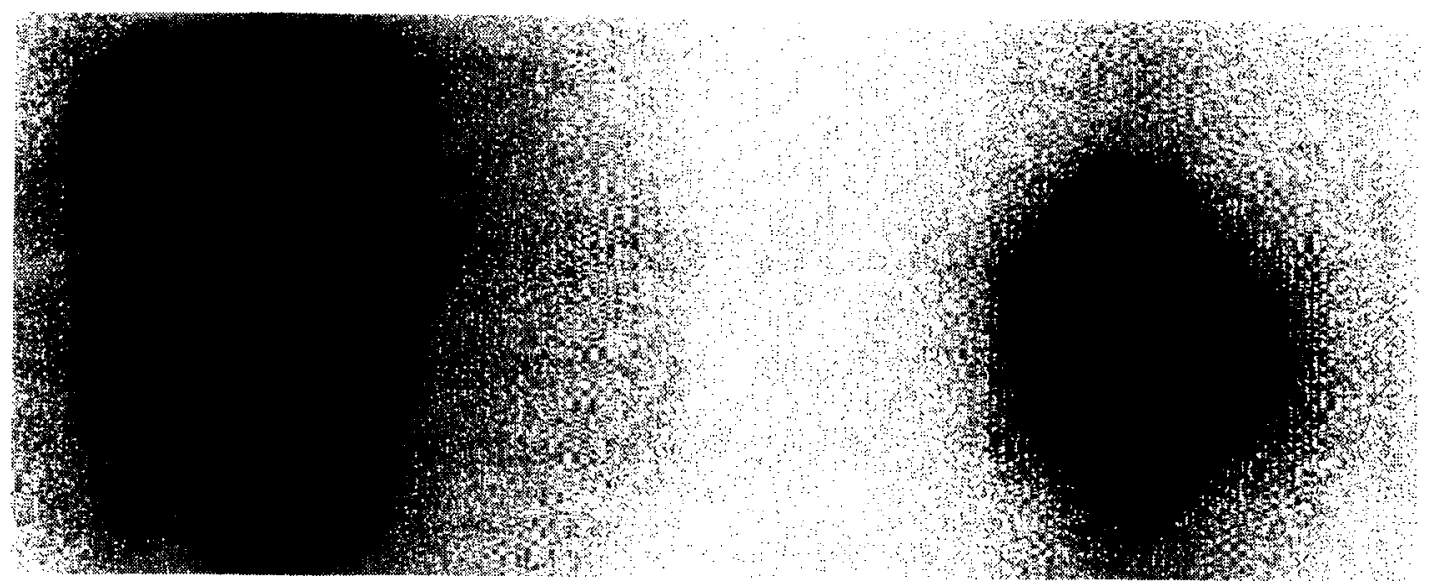

Fig. 5. The effect of phaseconjugation on the MOPA output, left: without, right: with.

realized. Higher reflectivities are expected at higher input powers and improved beam quality at the focusing cells. A picture of the effect of the phaseconjugation on the MOPA output is shown in Fig. 5. Both cells are all quartz cells with AR-coated windows and contain HPLC-grade Freon-113. Freon is a powerful solvent and polymer type materials (glues, grease and o-rings) must be avoided to maintain high purity and therefore low absorption. Absorption would cause thermal scattering and breakdown in the fluid which destroys the phase conjugation. Also the reflectivity of the cells would suffer heavily from absorption because it increases the threshold for scattering.

\section{Results and discussion}

As can be seen in Table 1 the single pass gain increases with repetition rate. This is due to the temperature of the pumping diodes that cannot be raised to the right temperature for low repetition rate in our cooling setup. At higher repetition rate the temperature Table I

Input-, double pass -, and output-power for various repetition rates

\begin{tabular}{cccc}
\hline $\begin{array}{c}\text { Rep. } \\
\text { rate (Hz) }\end{array}$ & $\begin{array}{c}\text { Input } \\
\text { (W) }\end{array}$ & $\begin{array}{c}\text { Double } \\
\text { pass (W) }\end{array}$ & $\begin{array}{c}\text { Output } \\
\text { (W) }\end{array}$ \\
\hline 100 & 0.29 & 1.6 & 2.3 \\
200 & 0.58 & 4.2 & 4.9 \\
300 & 0.64 & 6.4 & 15 \\
400 & 0.70 & 7.3 & 20 \\
\hline
\end{tabular}

increases and the wavelength radiated by the diodes shifts into the Nd:YAG absorption. The single pass gain increases and although the oscillator energy per pulse decreases the energy that impinges on the Brillouin cell sharply increases. The Brillouin reflectivity depends non-linearly on the intensity and therefore the reflected energy increases and the output increases. At powers above $20 \mathrm{~W}$, optimum overlap with the Nd:YAG absorption can be obtained by adjusting the cooling water temperature. Since the injection seeding of the oscillator is not stabilised occasional breakdown (less than 1 per minute) was observed for badly seeded pulses. Otherwise the output was stable and showed excellent propagation properties. Using a selfreferencing interferometer [12] a phasefront flatness better then $\lambda / 5$ (limited by the interferometer optics) was measured in combination with a Gaussian intensity profile. This compares to a value for $M^{2}$ of less then 1.2. The amplifier has been used up to $1 \mathrm{kHz}$ and shows the expected gain. In this setup the oscillator is limiting the system. With a new zig-zag slab oscillator we hope to reduce the lensing and the thermal depolarization losses. This oscillator will be a ring oscillator to enhance the extraction efficiency at single longitudinal operation. With reasonable oscillator output at 1 $\mathrm{kHz}$ we expect to reach $50 \mathrm{~W}$ for the MOPA system.

\section{References}

[1] D.A. Rockwell, IEEE J. Quantum Electron. QE-24 (1988) 1124. 
|2| N.F. Andreev, E. Khazanov and G.A. Pasmanik, IEEE J. Quantum Electron. QE-28 (1992) 330.

(3) D.S. Sumida, D.C. Jones and D.A. Rockwell, IEEE J. Quantum Electron. QE-30 (1994) 2617.

14| C.B. Dane, L.E. Zapata, W.A. Neuman, M.A. Norton and L.A. Hackel, IEEE J. Quantum Electron. QE-31 (1995) 148.

15| C.B. Dane, W.A. Neuman and L.A. Hackel, Optics Lett. 17 (1992) 1271.

|6| C.L. Tang, J. Appl. Phys. 37 (1966) 2945.

17| R. Menzel and H.J. Eichler, Phys. Rev. A 46 (1992) 7139.

|8| J. Richards and A. McInnes, Optics Lett. 20 (1995) 371.

19| D.R. Walker, C.J. Flood, H.M. van Driel, U.J. Greiner and H.H. Klingenberg, Optics Lett. 19 (1994) 1055.
[10] H.P. Godfried, E.A.J.M. Bente and H.L. Offerhaus, OSA Proc. on Advanced Solid State Lasers, eds. B.H.T. Chai and S.A. Payne (Optical Society of America, Washington, DC, 1995) to be published.

[11] G.J. Crofts, M.J. Damzen and R.A. Lamb, J. Opt. Soc. Am. B 8 ( 1991) 2282.

[12] C.B. Edwards, P. Lee and M. Tatarakis, SPIE Proceedings, Vol. 2375, Beam Control, Diagnostics, Standards, and Propagation, eds. W. Lindsay, A. Giesen, D.H. Leslie and H. Weichel (SPIE, Bellingham WA, 1995) p. 254. 